\title{
Maternal-Fetal Disposition and Metabolism of Retrorsine in Pregnant Rats
}

\author{
Xia Li, ${ }^{1}$ Xiaojing Yang, ${ }^{1}$ E Xiang, Jinyuan Luo, Shuaikai Qiu, Yan Fang, Li Zhang, Yu Guo, \\ Jiang Zheng, and Hui Wang
}

Department of Pharmacology, School of Basic Medical Science, Wuhan University, Wuhan (X.L., E.X., J.L., S.Q., Y.F., Y.G., H.W.); and Hubei Provincial Key Laboratory of Developmentally Originated Disease, Wuhan University, Wuhan (Y.G., H.W.); Wuya College of Innovation, Shenyang Pharmaceutical University, Shenyang, Liaoning (X.Y., J.Z.); and State Key Laboratory of Functions and Applications of Medicinal Plants, Key Laboratory of Pharmaceutics of Guizhou Province, Guizhou Medical University, Guiyang, Guizhou (J.Z.); Department of Pathology, School of Basic Medical Science, Wuhan University, Wuhan (L.Z.), China

Received October 31, 2017; accepted January 17, 2018

\section{ABSTRACT}

Pyrrolizidine alkaloids (PAs) are extensively synthesized by plants, are commonly present in herbs and foodstuffs, and exhibit hepatotoxicity requiring metabolic activation by cytochrome P450 3A to form the electrophilic metabolites-pyrrolic esters. PAs also cause embryo toxicity, but the metabolic profiles of PAs in fetus and placenta have been far from clear. In this study, we determined the basal metabolic activation of retrorsine (RTS) in rat maternal liver, placenta, and fetal liver in vitro and examined the fetal toxicity and bioactivation of RTS in vivo. Detection of microsomal RTS metabolites in vitro showed that the basal metabolic activity of fetal liver and placenta to RTS was much weaker than that of maternal liver. In addition, a higher rate of pyrrolic ester formation was found in normal male fetal liver compared with that of female pups. In vivo exposure to RTS caused fetal growth retardation, as well as placental and fetal liver injury. Little difference in serum RTS was observed in dams and fetuses, but the content of pyrrole-protein adduction in the fetal liver was much lower than that in maternal liver, which was consistent with basal metabolic activity. Unexpectedly, compared with basal metabolism in fetal liver, exposure to RTS during middle and late pregnancy caused an opposite gender difference in RTS metabolism and CYP3A expression in the fetal liver. For the first time, our study showed that RTS can permeate the placenta barrier and entering fetal circulation, whereas the intrauterine pyrrolic metabolite was generated mainly by fetal liver but not transported from the maternal circulation. Induction of CYP3A by RTS was gender-dependent in the fetal liver, which was probably responsible for RTS-induced fetal hepatic injury, especially for female pups.

\section{Introduction}

Pyrrolizidine alkaloids (PAs) are a type of defense constituents with similar chemical structures and that are wildly present in plants. PAs have been attracting attention because of their severe hepatotoxicity and wide range of sources placing humans at a high risk for exposure. Approximately $3 \%$ of flowering plants contain toxic PAs, and 660 kinds of PAs and their $N$-oxide metabolites have been found in more than 6000 plants in worldwide (Smith and Culvenor, 1981; Radominska-Pandya 2010). Many foodstuffs, such as milk, honey, eggs, tea, and plant spices, were reportedly contaminated by PAs (Huybrechts and Callebaut, 2015). Research showed that unsaturated PAs exhibited notable toxicity after metabolic activation mainly by cytochrome P450 (P450) 3A to form electrophilic intermediates (pyrrolic esters), which attack a variety of important intracellular

This work was funded by grants from the National Natural Science Foundation of China [Grant 81473290, 30901835, and 81430086], Hubei Province Health and Family Planning Scientific Research Project [Grant WJ2017M005], and the Fundamental Research Funds for the Central Universities [Grant 2042017kf1032].

${ }^{1} \mathrm{X}$.L. and X.Y. contributed equally to this work.

https://doi.org/10.1124/dmd.117.079186. nucleophilic substances (e.g., DNA and protein) (Yang et al., 2001; Ruan et al., 2015). Pyrrolic esters can be detoxified by conjugating with glutathione (GSH), and excessive PA exposure may cause depletion of GSH and adduction of DNA and protein, lead to hepatocyte apoptosis and death, and result in hemorrhagic necrosis, liver macrocytosis and vein occlusion (Zhou et al., 2007).

Besides hepatic damage, PAs can cause embryo toxicity. Hepatic sinusoidal obstruction syndrome was observed in a newborn infant from a mother who drank herbal tea during gestation, and the tea contained a detectable level of PA seconine (Roulet et al., 1988). Animal studies reported fetal deaths, weight loss, and teratogenesis in pregnant rats receiving PAs from early pregnancy (Bhattacharyya 1965; Panter et al., 1998; Medeiros et al., 2000). Our early work demonstrated that prenatal exposure to PAs alone or with lipopolysaccharide led to fetal mortality and preterm in mice (Guo et al., 2013); but many aspects of PA-induced embryo toxicity remain unclear. For instance, PAs are presumed to enter fetal blood circulation through the placental barrier and associate with embryo toxicity; however, no experimental evidence is available up to date (Sundareson 1942).

In addition, although early research showed that human placenta expressed P450s 2C, 2D6, and 3A7 during pregnancy (Hakkola et al., 1996a), and rat placenta also contained a variety of P450 isoforms,

ABBREVIATIONS: CE, collision energy; CXP, collision cell exit potential; DABA, 4-dimethylaminobenzaldehyde; DP, declustering potential; GAPDH, glyceraldehyde phosphate dehydrogenase; GD, gestation day; GSH, glutathione; IS, internal standard; PAs, pyrrolizidine alkaloids; P450, cytochrome P450; RTS, retrorsine. 
such as CYP3A1 (Ejiri et al., 2003), whether CYP3A protein in placenta has function to catalyze xenobiotics metabolism is controversial (Hakkola et al., 1996b; Maezawa et al., 2010; Fabian et al., 2016). Most importantly, whether fetal liver has the ability to metabolize PAs needs to be verified so that we can evaluate the contribution of fetal metabolism to PA-induced embryotoxicity. Although it has been reported that hepatic CYP3A protein was detectable in both human and rat fetuses (Wyde et al., 2005; Hines, 2008), there was still lack of evidence that toxic metabolites of PAs can be produced by fetal liver.

Furthermore, the hepatotoxicity of PAs was reported to show gender difference. Lin and coworkers found that metabolic activation of clivorine, a naturally occurring pyrrolizidine alkaloid, took place more in adult male rats than in female rats (Lin et al., 2002, 2003, 2007), which indicates that male rats may be more susceptible to PA-induced toxicity. In fetus, androgens secreted by developing testis may induce gender-specific patterns of metabolic enzyme activity in liver (O'Shaughnessy et al., 2011). As such, we assumed that PA metabolism in fetal liver might also exhibit a gender difference.

Retrorsine (RTS, Scheme 1) is a commonly existing and extensively studied PA. Like most PAs, it is also activated by CYP3As in rats (Wang et al., 2005). The objectives of the present study were to determine the toxicity and metabolic activation of RTS in maternal-placental-fetal unit of rats and to examine the gender difference in RTS metabolism of rat fetuses. This study intended to seek solid evidence for metabolic toxicity of RTS in rat placenta and fetus and may allow us to understand more fully the embryotoxicity caused by RTS.

\section{Materials and Methods}

Reagents. RTS ( $\geq 98 \%)$, monocrotaline ( $\geq 98 \%)$, diazepam $(\geq 98 \%)$, glutathione (GSH, $\geq 98 \%$ ), 4-dimethylaminobenzaldehyde, $S$-hexylglutathione $(\geq 97 \%)$, silver nitrate, and NADPH were purchased from Sigma Chemical Co. (St. Louis, MO). Formic acid, trifluoroacetic acid, and perchloric acid were acquired from Fisher Scientific (Springfield, NJ). Goatanti CYP3A polyclonal antibody was obtained from Santa Cruz (catalog sc-30621; Dallas, TX). Mouse anti-glyceraldehyde phosphate dehydrogenase (GAPDH) antibody was purchased from Abclonal (catalog AC002; Wuhan, Hubei, China). All organic solvents were obtained from Fisher Scientific. Other reagents and solvents were either analytical or high-performance liquid chromatography grade.

Chemical Synthesis. Glutathione conjugates 35 (Scheme 1) were synthesized by reaction of dehydromonocrotaline preprepared with GSH, following the protocol we published earlier (Chen et al., 2016). Authentic compound $\mathbf{1 0}$ was prepared according to the protocol published in our early paper (Lin et al., 2011; Ruan et al., 2015).

Animals and Treatment. Specific pathogen-free (SPF) Wistar rats (female body weight: $200 \pm 20 \mathrm{~g}$, male body weight: $280 \pm 20 \mathrm{~g}$ ) were purchased from Experimental Center of Medical Scientific Academy of Hubei Province (China). The animals were allowed free access to food and water and maintained on a 12-hour light/dark cycle in a controlled temperature (20 \pm $2{ }^{\circ} \mathrm{C}$ ) room. All animal experimental procedures were performed in accordance with the Guidelines for the Care and Use of Laboratory Animals of the Chinese Animal Welfare Committee.

Two female rats were mated with one male overnight (from 7:00 PM to 7:00 AM the next morning). The day when a vaginal plug was found was designated as gestation day 0 (GD0). Half of the dams without any treatment were sacrificed on GD20; maternal liver, fetal liver, and placenta were harvested for microsome preparation through multistep centrifugation ( $200 \mathrm{~g}$ for 20 minutes, $9000 \mathrm{~g}$ for 20 minutes, and $10,500 \mathrm{~g}$ for 1 hour). The microsomes were stored in $-80^{\circ} \mathrm{C}$ for further basal metabolism assay and enzyme kinetic study.

The other half of the pregnant rats were intragastrically given vehicle or RTS at various doses from GD9 to GD20. Animals in the control group were treated with vehicle. Rats in groups RTS5, RTS10, and RTS20 were administrated with RTS at 5,
10 , or $20 \mathrm{mg} / \mathrm{kg}$, once per day, respectively. RTS was suspended in water, followed by dropwise addition of dilute $\mathrm{HCl}$ solution until the alkaloids were dissolved. The resulting acidic solution was neutralized by the addition of a $\mathrm{NaOH}$ solution. All the dams were euthanized by ether anesthesia or decapitation on GD20, 2 hours after RTS administration. Pup body weights were recorded, and simultaneously, maternal and fetal serum and livers as well as placenta were collected for further analysis.

Morphologic Examination. For RTS-treated rats, maternal and fetal livers, as well as placenta, were fixed with $10 \%$ neutral formalin. Paraffin sections (the thickness was $5 \mu \mathrm{m}$ ) were prepared and then stained with H\&E. Histomorphologic changes of these tissues were evaluated under light microscopy, and photomicrographs were captured using Photo Imaging System (TE2000; Nikon, Japan). The tissue slides were obtained from at least three dams per group, and the results were verified independently by four authors (X.L., J.L., Y.G., and L.Z.; L.Z. is a pathologist with expertise in morphologic examination).

Determination of Serum RTS in Dams and Fetuses. Serum levels of RTS were estimated by a reported method with some modification described by our laboratory (Yang et al., 2017). Aliquots ( $20 \mu \mathrm{l})$ of serum sample were mixed with $50 \mu \mathrm{l}$ of acetonitrile containing monocrotaline as the internal standard (IS). The resulting mixtures were vortex-mixed and centrifuged at 16,000 $\mathrm{g}$ for 10 minutes to remove the precipitated protein. The resultant supernatants were diluted with three volumes of water and then subjected to an LC-MS/MS system for analysis.

Determination of Pyrrole-Protein Adduction in Livers of Maternal and Fetal Rats. Liver tissues $(0.2 \mathrm{~g})$ were homogenized in $2 \mathrm{ml}$ of phosphate buffer $(\mathrm{pH} 7.4,100 \mathrm{mM})$, followed by centrifuging at $9000 \mathrm{~g}$ for 10 minutes. The supernatants were used for the determination of hepatic protein adduction levels by the method established in our laboratory (Lin et al., 2011). Briefly, each liver sample $(100 \mu \mathrm{l})$ was mixed with $500 \mu \mathrm{l}$ of acetone, vortexed, and centrifuged at $900 \mathrm{~g}$ for 5 minutes. After being washed with $500 \mu \mathrm{l}$ of absolute ethanol, the resulting pellets were reconstituted in $500 \mu \mathrm{l}$ of freshly prepared $2 \%$ silver nitrate ethanol solution containing 5\% trifluoroacetic acid and were shaken for 30 minutes at room temperature. After centrifugation at $19,000 \mathrm{~g}$ for 10 minutes, the resulting supernatants were reacted with 4-dimethylaminobenzaldehyde (v/v 4:1) in ethanol containing $1 \%$ perchloric acid at $55^{\circ} \mathrm{C}$ for 10 minutes. To each sample was added $10 \mu \mathrm{l}$ of IS (diazepam, $1.25 \mu \mathrm{g} / \mathrm{ml}$ ) and the resultant mixtures were injected into an LC-MS/MS system for analysis. The chemical structure of the molecule (compound 10) detected by LC-MS/MS is shown in Scheme 1.

Enzyme Kinetic Study. The preparation of rat liver and placenta microsomes were carried out as described by our laboratory (Lin et al., 2007). For enzyme kinetic studies, the incubation mixtures consisted of $0.25 \mathrm{mg} / \mathrm{ml}$ of microsomal protein (including maternal and fetal rat liver microsomes and placenta microsomes), $3.2 \mathrm{mM} \mathrm{MgCl} 2,10 \mathrm{mM} \mathrm{GSH}, 100 \mathrm{mM}$ potassium phosphate buffer (pH 7.4), RTS (100-8000 $\mu \mathrm{M}$ ), and 1.0 mM NADPH. The final volume of each sample was $200 \mu \mathrm{l}$, and the total organic concentration of each incubation mixture was $<1 \% \mathrm{v} / \mathrm{v}$ acetonitrile. The reaction was stopped by the addition of $200 \mu \mathrm{l}$ of ice-cold acetonitrile after 20 minutes' incubation at $37^{\circ} \mathrm{C}$. For comparison, incubations were performed with optimized microsomal protein concentration and incubation time in their linear ranges. The resulting mixtures were vortex-mixed and centrifuged at 19,000 $\mathrm{g}$ for 10 minutes. The supernatants were diluted with equal volumes of water and then analyzed by the LC-MS/MS system. All experiments were performed in triplicate. In the incubation system, the catalytic capabilities of the enzymes were evaluated by the MS peak areas of pyrrole-GSH conjugates 4 and 5 (integrated). GraphPad Prism version 5.04 (GraphPad Software, San Diego, CA) was used in the statistical analyses of enzyme kinetic parameters.

LC-MS/MS Method. The quantifications of RTS and its metabolite were performed on an AB SCIEX Instruments 5500 triple quadrupole mass spectrometry (Applied Biosystems, Foster City, CA) equipped with an Agilent 1260 Series Rapid Resolution LC system. Data were analyzed using Applied Biosystems/SCIEX Analyst software (version 1.6.2). Samples were analyzed by multiple-reaction monitoring scanning in positive ion mode. The optimized MS instrument parameters were as follows: ion spray voltage was set at $5500 \mathrm{~V}$, and ion source temperature was set at $650^{\circ} \mathrm{C}$. Curtain gas, gas 1 , and gas 2 were 20,50 , and $50 \mathrm{psi}$, respectively. The characteristics of ion pairs [declustering potential (DP), collision energy (CE), and collision cell exit potential (CXP)], and respective LC conditions were listed as follows.

For analysis of RTS, the chromatographic separation was achieved on a Kinetex HILIC $(50 \times 2.1 \mathrm{~mm}, 2.6 \mu \mathrm{m})$ column (Phenomenex, Torrance, CA) 
protected by a Security-Guard $(4.0 \times 3.0 \mathrm{~mm}, 5.0 \mu \mathrm{m}) \mathrm{C} 18$ column (Phenomenex). The mobile phase was composed of acetonitrile (A)/ammonium acetate $(5 \mathrm{mM}, \mathrm{B})$ with a gradient elution as follows: 0-3.5 minutes, $15 \% \mathrm{~B} ; 3.5-$ 4.5 minutes, $15 \%-60 \%$ B ; $4.5-5.5$ minutes, $60 \%$ B; $5.5-6.5$ minutes, $60 \%-15 \%$ $\mathrm{B}$; and $6.5-11$ minutes, $15 \% \mathrm{~B}$. Flow rate was set at $0.4 \mathrm{ml} / \mathrm{min}$. The characteristic ion pair (DP, CE, and CXP) for RTS and monocrotaline (IS) were $\mathrm{m} / \mathrm{z}$ $352.1 \rightarrow 119.9(100,48,10)$ and $\mathrm{m} / \mathrm{z} 326.1 \rightarrow 120.2(270,34,13)$.

For analysis of pyrrole-protein adduction, the chromatographic separations were achieved on a CAPCELL PAK UG-C18 column $(250 \times 4.6 \mathrm{~mm}, 5.0 \mu \mathrm{m})$ (Shiseido, Tokyo, Japan) protected by a Security-Guard $(4.0 \times 3.0 \mathrm{~mm}, 5.0 \mu \mathrm{m})$ C18 column (Phenomenex). Mobile-phase systems containing acetonitrile with $0.1 \%$ formic acid (A) and water with $0.1 \%$ formic acid (B) were used with a gradient elution as follows: $0-2$ minutes, $70 \%-70 \% \mathrm{~B} ; 2-10$ minutes, $70 \%-10 \%$ $\mathrm{B} ; 10-12$ minutes, $10 \%-10 \% \mathrm{~B} ; 12-14$ minutes, $10 \%-70 \% \mathrm{~B}$; and 14 17 minutes, $70 \%-70 \% \mathrm{~B}$. Flow rate was set at $0.8 \mathrm{ml} / \mathrm{min}$. The characteristic ion pairs (DP, CE, and CXP) were $m / z, 341.0 \rightarrow 252.1(100,40,10)$ for pyrroleprotein adduction and $\mathrm{m} / \mathrm{z}, 285.1 \rightarrow 193.5(101,46,10)$ for diazepam (IS), respectively. For determination of pyrrole-GSH conjugates, the chromatographic separations were also performed on the CAPCELL PAK UG-C18 column system. The mobile phase system, including acetonitrile with $0.1 \%$ formic acid (A) and water with $0.1 \%$ formic acid (B), was used with a gradient elution as follows: $0-$ 2 minutes, $90 \%-90 \% \mathrm{~B} ; 2-10$ minutes, $90 \%-10 \% \mathrm{~B} ; 10-12$ minutes, $10 \%-$ $10 \% \mathrm{~B} ; 12-14$ minutes, $10 \%-90 \% \mathrm{~B}$; and $14-17$ minutes, $90 \%-90 \% \mathrm{~B}$. Flow rate was $0.8 \mathrm{ml} / \mathrm{min}$. The parameters of ion pairs (DP, CE, and CXP) were $\mathrm{m} / \mathrm{z}$ $425.0 \rightarrow 118.0(100,30,13)$ for GSH conjugate and $\mathrm{m} / z, 392.2 \rightarrow 246.3(86,24,5)$ for $S$-hexylglutathione (IS), respectively.

Western Blot. Protein extraction from RTS-treated fetal liver was carried out as described in the protocol of kit (Beyotime Biotechnology, Nantong, Jiangsu, China), and maternal liver microsomes were used as the positive control. Protein extracts were separated in 5\%-10\% SDS-PAGE and electrophoretically transferred onto polyvinylidene fluoride membranes. After blocking with $5 \%$ nonfat milk in phosphate buffer, CYP3A proteins were determined by immunoblot using goat anti-CYP3A antibody, and GAPDH proteins were determined as housekeeping proteins using mouse anti-GAPDH antibody. Membranes were then incubated with secondary antibodies conjugated with horseradish peroxidase, and specific protein bands were visualized by chemiluminescence detection, according to the manufacturer's protocol (Bridgen, Beijing, China).

Statistical Analysis. The data were analyzed using Statistical Package for the Social Sciences version 17.0 software (SPSS Inc, Chicago, IL). Unless stated otherwise, data are presented as mean \pm S.E.M. Two-way analysis of variance was performed to determine the interaction between gender (female and male) and group (control and RTS). Comparison of multiple means with analysis of variance, after the equal check of variance, and individual group means were compared using Bonferroni's test. $P<0.05$ was considered significantly different.

\section{Results}

Basal Metabolism of RTS in Rat Maternal-Placental-Fetal Unit In Vitro. RTS was metabolized to a pyrrolic ester via oxidation reaction catalyzed by $\mathrm{P} 450$ enzymes, and the reactive metabolite sequentially reacted with GSH to form the corresponding GSH conjugates, and the formation of the GSH conjugates most likely represented the generation of the pyrrolic ester. As shown in Fig. 1, microsomes of normal maternal and fetal livers, as well as placenta, were able to catalyze the metabolic activation of RTS, but the efficacies varied. Significantly higher metabolic activation of RTS was observed in maternal liver microsomes compared with that of the microsomes obtained from fetal liver and placenta $(P<0.01)$, whereas no distinct difference was seen in the formation of pyrrole-GSH conjugates in fetal liver and placenta microsomes incubated with RTS.

Efficiency to Bioactivate RTS in Maternal and Fetal Liver Microsomes. Michalis-Menten plots and enzyme kinetic parameters are shown in Fig. 2 for the biotransformation of RTS in normal maternal and fetal livers. The $K_{\mathrm{m}}$ and $V_{\max }$ values were calculated based on the production of GSH conjugates 4 and $\mathbf{5}$ (integrated). $V_{\max }$ value was greater in maternal liver microsomes than that in fetal liver microsomes,

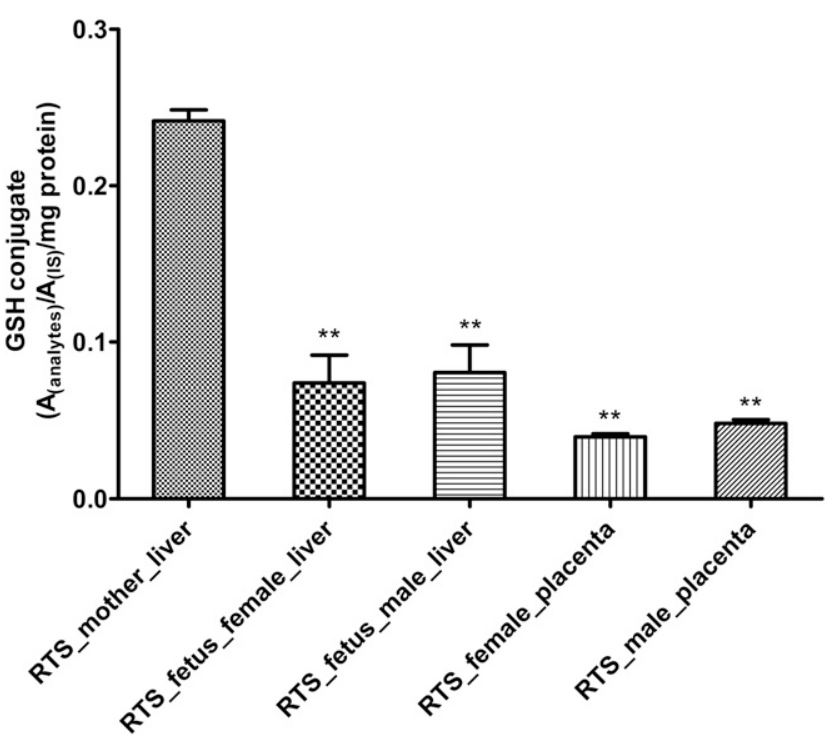

Fig. 1. Pyrrole-GSH conjugates generated in microsomes treated with RTS. Microsomes were prepared from maternal and fetal livers, as well as placenta in intact pregnant rats on GD 20. RTS $(1 \mathrm{mM})$ was incubated with individual microsomes and NADPH in the presence of GSH. Data are shown as mean \pm S.E.M., $n=3$. $* P<0.05 ;{ }^{*} * P<0.01$ vs. maternal liver, ${ }^{\#} P<0.05$ vs. RTS treatment According to two-way analysis of variance (ANOVA) analysis, there was no interaction between gender and RTS treatment.

and the $K_{\mathrm{m}}$ value of maternal liver microsomes was much less than that of liver microsomes prepared from pups. Maternal hepatic $C L_{\text {int }}$, calculated by $V_{\max } / K_{\mathrm{m}}$, was 21.9 -fold higher than that of female fetal liver microsomes and was 10.8-fold higher than that of male fetal liver microsomes, respectively. Besides, male fetal microsomes exhibited a better catalytic activity than female to bioactivate RTS.

Fetal Toxicity of RTS. As shown in Fig. 3, no distinct difference was noted in fetal weight between control and low doses of RTS (5 and $10 \mathrm{mg} / \mathrm{kg})$ groups; however, a significant decrease $(P<0.01)$ in the body weight of pups was observed in dams treated with the high dose $(20 \mathrm{mg} / \mathrm{kg}$ ) compared with that of the control animals. There was no gender difference in fetal body weight in any group.

Little pathohistologic changes in mother liver were detected in the low groups $(5.0$ and $10 \mathrm{mg} / \mathrm{kg}$ ) (Fig. 4, B and C), whereas swelling and vacuolization of hepatocytes were observed in dams treated with the high dose (20 mg/kg, Fig. 4D). Normal rat fetal liver consisted of immature hepatocytes, multinucleated giant cells, and vacuolar parenchyma cells (Fig. 4, E and I). An increased number of parenchyma cells and loss of hepatocytes were observed in the pups of animals given the high dose (Fig. 4, H and L), especially females, and increased vacuolar cells were seen, even in the group that had $10 \mathrm{mg} / \mathrm{kg}$ (Fig. 4G). Rat placenta comprises decidua (maternal placenta area), junctional zones, and labyrinthine zones (fetal placenta area). Compared with the control group, RTS exposure caused morphology disorder in placental tissue, especially for the high-dose group (20 mg/kg, Fig. 4, N-P and R-T). Necrotic glycogen cells in junctional zones were observed, accompanied by cysts and congestion. Additionally, a loss of cells in the labyrinthine zone was found, along with irregular arrangement. Placentas of both genders showed similar morphologic changes.

Levels of RTS in Rat Maternal and Fetal Serum. Elevated serum RTS was observed in both dams and fetuses along with dose increase. The level of RTS in fetal sera was proportional to that in maternal sera, and no difference was noted in the serum RTS between two genders of pups (Fig. 5), suggesting that most RTS circulated in maternal blood permeated rat placenta and entered fetal blood flow. 

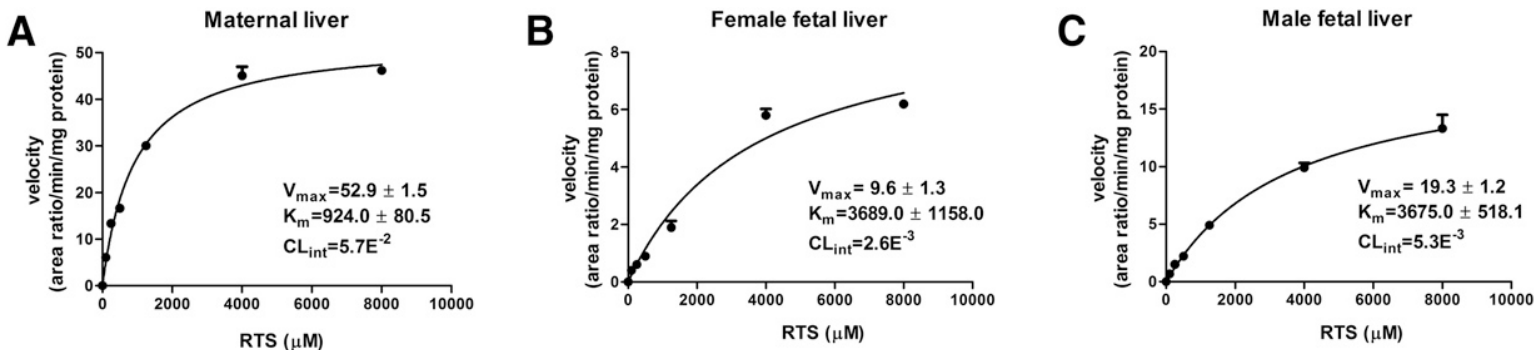

Fig. 2. Michaelis-Menten kinetic assessment of RTS in rat maternal and fetal liver microsomes. Microsomes were incubated with RTS at various concentrations (100-8000 $\mu \mathrm{M}$ ) for 20 minutes. (A) Maternal liver microsomes; (B) female fetal liver microsomes; (C) male fetal liver microsomes. The following were the units for enzyme kinetic parameters: $V_{\max }$ (micrograms per minute per milligram of protein), $K_{\mathrm{m}}(\mu \mathrm{M})$ and $C L_{\text {int }}$ (liters per minute per milligram of protein). Data are shown as mean \pm S.E.M., $n=3$.

Pyrrole-Protein Adduction in Maternal-Placental-Fetal Unit In Vivo. The levels of hepatic pyrrole-protein adduction in mice given RTS were assessed by chemical derivatization of the protein adducts to compound 10 (Scheme 1). As shown in Fig. 6, pyrrole-protein adduction was detected in maternal and fetal liver, as well as placenta from dams administered RTS. The contents of pyrrole-protein adducts formed in the placenta of female pups was about $36.7 \%, 45.2 \%$, and $69.9 \%$ of maternal hepatic pyrrole-protein adduction in groups 5.0, 10, and $20 \mathrm{mg} / \mathrm{kg}$, respectively. The levels of pyrrole-protein adduction in male fetal placenta were similar to those in the placenta of female pups. No gender difference in placental pyrrole-protein adduct content was seen.

RTS-induced protein adduction in fetal liver was much less than that in maternal liver and placenta. Although fetal hepatic pyrrole-protein adduction increased in a dose-dependent manner, the levels of pyrroleprotein adduction in female and male pups exposed to RTS at $20 \mathrm{mg} / \mathrm{kg}$ were only $9.0 \%$ and $4.8 \%$ of maternal hepatic pyrrole-protein adduction, respectively $(P<0.01)$. According to two-way analysis of variance analysis, there was no interaction between gender and RTS treatment, but there was a significant gender difference of pyrrole-protein adduction content in fetal liver. The levels of pyrrole-protein adduction in female fetal liver were higher than that in male fetal liver $(P<0.01)$.

Effect of RTS Treatment on CYP3A Expression in Fetal Liver. Expression of hepatic CYP3A was higher in male fetuses than that in female pups from dams treated with vehicle $(P<0.01$, Fig. 7). There was an interaction between fetal gender and RTS treatment. Prenatal RTS exposure significantly induced CYP3A expression in female fetal liver $(P<0.01)$, whereas downregulated CYP3A protein content was observed in male fetal liver $(P<0.01)$.

\section{Discussion}

Early study demonstrated that CYP3A enzyme was responsible for metabolic activation of RTS (Wang et al., 2005). To determine the role of fetal hepatic and placental CYP3A in the bioactivation of RTS and RTS-induced fetal toxicity, we assessed the basal metabolic activity of CYP3A in fetal liver and placenta obtained from normal pregnant rats on GD 20. It is evident that rat fetal liver and placenta were capable of catalyzing RTS metabolism (Fig. 1), although the basal catalytic efficiency of fetal CYP3A was lower than that in maternal liver. In addition, the results showed that basal metabolic efficiency of RTS in male fetal liver was higher than that in females, which is consistent with previous reports of gender difference in CYP3A expression and PA metabolic activation in adult rats (Lin et al., 2002, 2003, 2007).

To understand more fully the relationship between RTS-induced fetal toxicity and in situ RTS metabolic activation in placenta and fetal liver, we established a prenatal RTS-exposure rat model and started with investigation of changes in fetal body weight, as well as in hepatic and placental morphology in prenatal RTS-exposed rats. Prenatal exposure to RTS at $20 \mathrm{mg} / \mathrm{kg}$ during the second and third trimester led to a reduction of rat fetal body weight, which verifies previous reports that PA induced developmental retardation in rats (Peterson and Jago, 1980; Medeiros et al., 2000; Sandini et al., 2014). Remarkable paramorphia of placental trophoblast and fetal hepatocytes were observed in animals treated with RTS at $20 \mathrm{mg} / \mathrm{kg}$ (Fig. 4). Developing liver appeared to be more susceptible to this reactive hepatotoxicant, manifested by significant pathologic changes in fetal liver but slightly swelling of maternal hepatocyte (Fig. 4). Then we examined the parent RTS and pyrroleprotein adduction in utero. It has been speculated that PA was able to permeate placenta (Sundareson, 1942), but no experimental evidence was available in support of the speculation until our study. The level of RTS in fetal sera was similar to the level in maternal sera, especially for the high-dose group (Fig. 5). The barrier role of ATP-binding cassette transporters, especially P-glycoprotein, in placenta, was emphasized by Lankas et al. (1998), but Tu et al. reported that RTS is a weak substrate for P-glycoprotein (Tu et al., 2014), which we think may be why RTS entered the rat fetus through the placenta barrier almost without obstacle.

Pyrrolic esters are the main toxic metabolites of PAs. Because of their electrophilic reactivity, pyrrolic esters attack nucleophilic sites of protein to form pyrrole-protein adduction after being generated from parent PAs in situ, and the protein modification may trigger the hepatotoxicity of PAs. A considerable amount of pyrrole-protein adducts was observed in the placenta in rats prenatally exposed to RTS. Although we confirmed metabolic activation of RTS in placenta in vitro, we cannot exclude the

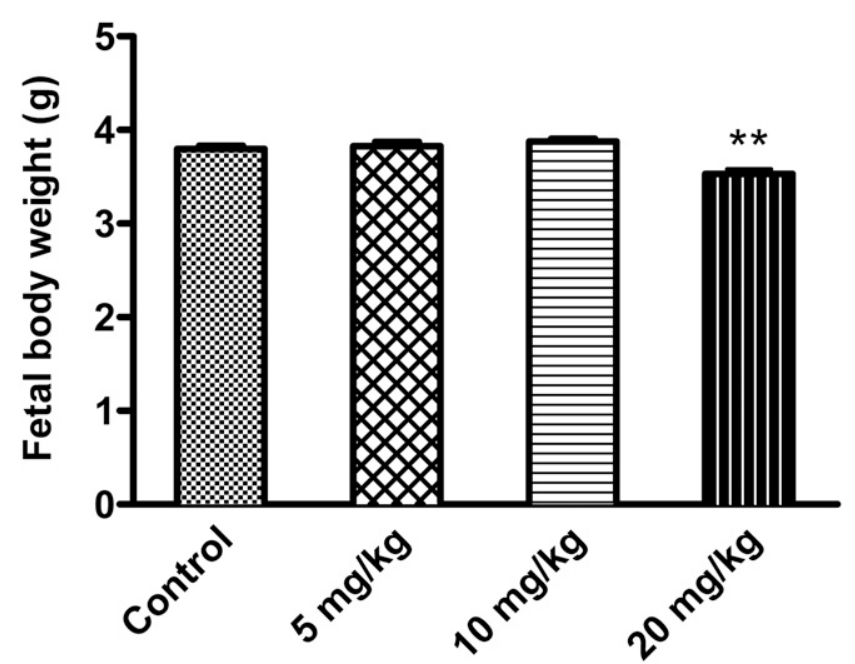

Fig. 3. Body weight of rat pups prenatally exposed to RTS. Pregnant rats were treated with RTS $(5.0-20 \mathrm{mg} / \mathrm{kg}$ ) from GD 9 to 20 . Data are shown as mean \pm S.E.M., $n=4-11$. $* * P<0.01$ vs. control. 


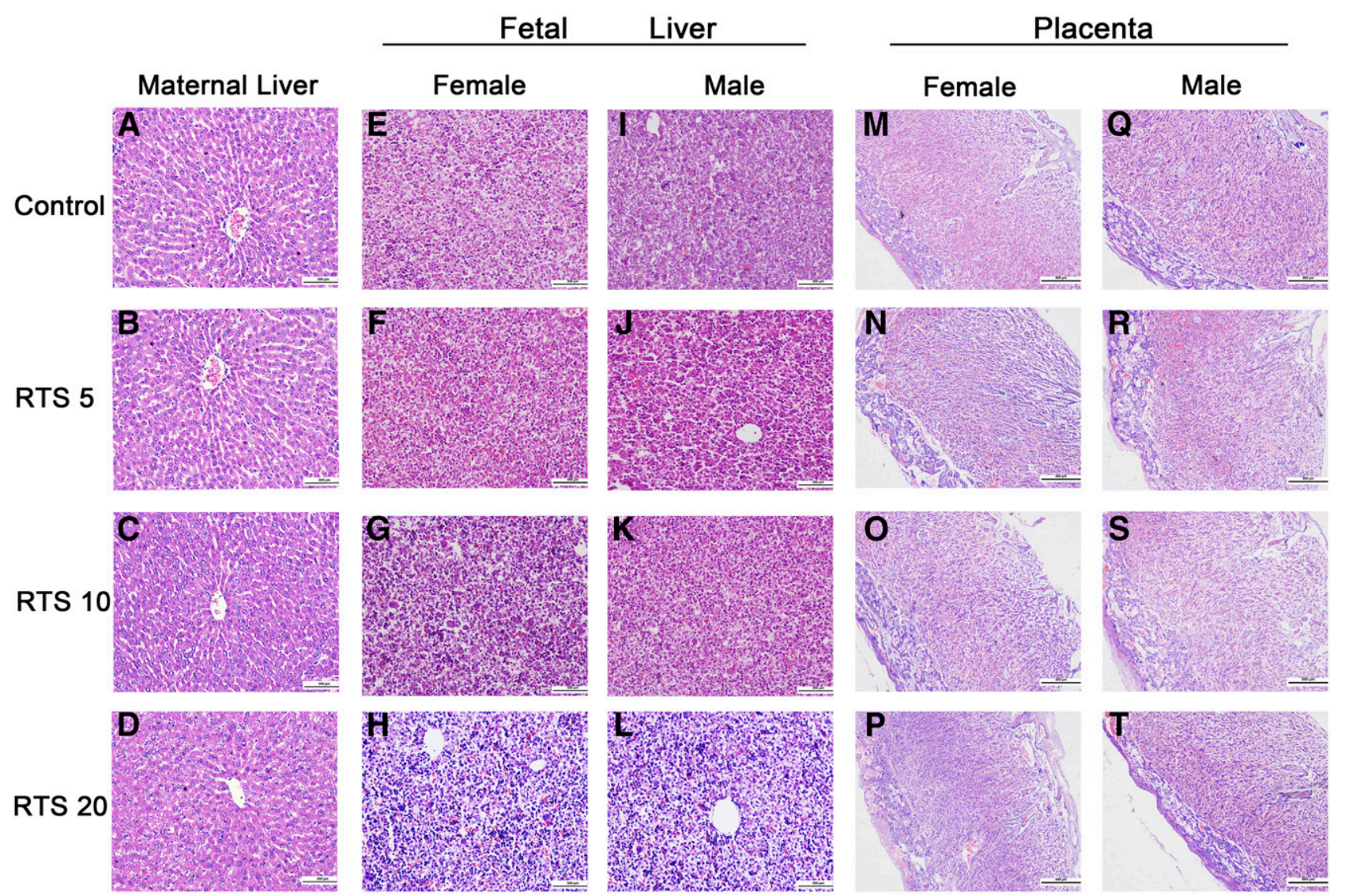

Fig. 4. Morphologic changes of maternal and fetal liver as well as placenta. Pregnant rats were treated with RTS (5.0-20 mg/kg) from GD 9-20. Magnification for maternal and fetal liver, 200×; the magnification for placenta, 40×. (A-D) Maternal liver; (E-H) female fetal liver; (I-L) male fetal liver; (M-P) female placenta; (Q-T) male placenta; (A, E, I, M and Q) control; (B, F, J, N and R) animals treated with $5 \mathrm{mg} / \mathrm{kg} \mathrm{RTS}$; (C, G, K, O and S) animals treated with $10 \mathrm{mg} / \mathrm{kg} \mathrm{RTS}$; (D, H, L, P and T) animals treated with $20 \mathrm{mg} / \mathrm{kg}$ RTS.

possibility that the reactive metabolite of RTS generated in maternal liver can be transported to placenta with blood flow and then exerted their toxicity by reacting with protein in placenta. The reason for this speculation is that pyrrolic ester-derived GSH conjugate in placental microsomes was only $10 \%$ of that in maternal liver microsomes in vitro, whereas similar levels of pyrrole-protein adduction were found in the placenta and maternal liver in vivo, suggesting that the reactive metabolite of RTS formed through maternal bioactivation probably participated, at least in part, in the metabolic injury of the placenta.

Pyrrole-protein adduction took place less in fetal liver than those in maternal liver and placenta in animals given RTS (Fig. 6); however, minor changes in histopathology were observed in maternal liver tissues. The explanation could be that the developing embryo has relatively low levels of CYP3A, which leads to the lower amount of pyrrole-protein

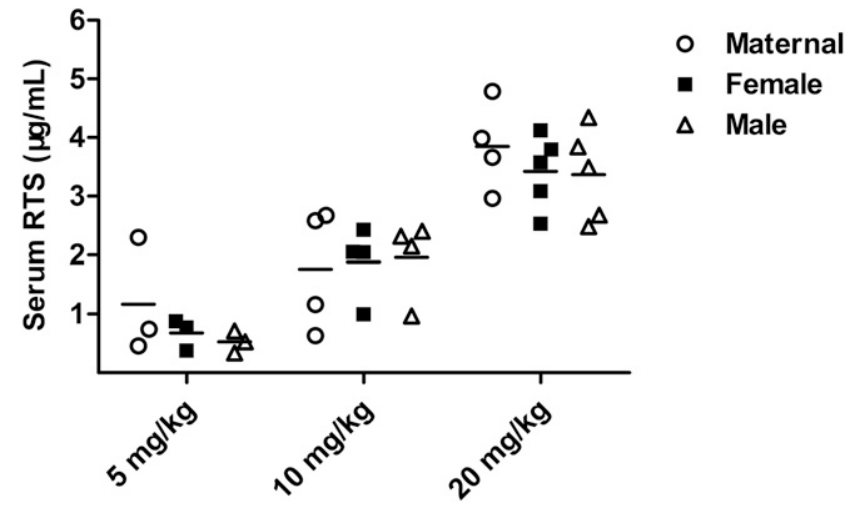

Fig. 5. Concentration of serum RTS in dams and fetuses. Pregnant rats were treated with RTS (5.0-20 mg/kg) from GD 9-20. Horizontal bars represent the average serum concentration of RTS in each group, $n=3-5$.

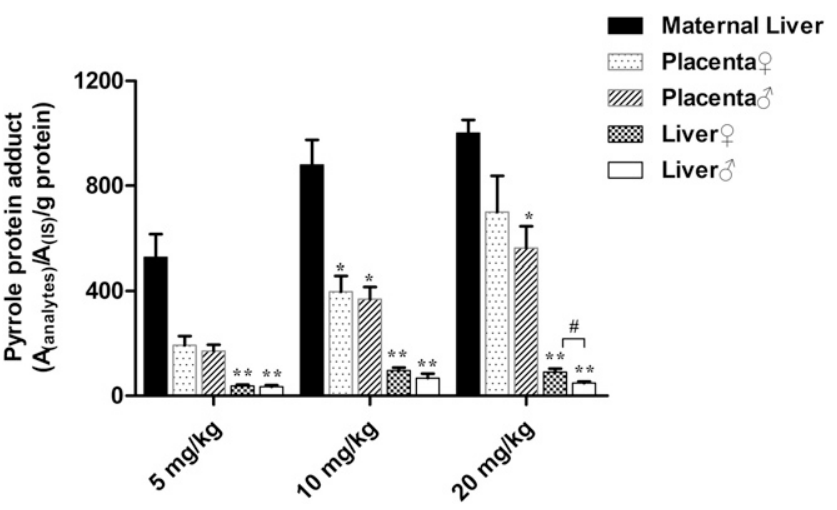

Fig. 6. Pyrrole-protein adduction in maternal-placental-fetal unit from pregnant rats treated with RTS. Pregnant rats were treated with RTS $(5.0-20 \mathrm{mg} / \mathrm{kg})$ from GD 9 20. Data are shown as mean \pm S.E.M., $n=3-5$. $* P<0.05 ; * * P<0.01$ vs. maternal liver; ${ }^{\# \#} P<0.01$ vs. female pup. According to two-way analysis of variance (ANOVA) analysis, there was no interaction between gender and RTS treatment. 


\section{Controlo RTS우 Control $\hat{o}$ RTS $\hat{\text { s }}$ control}

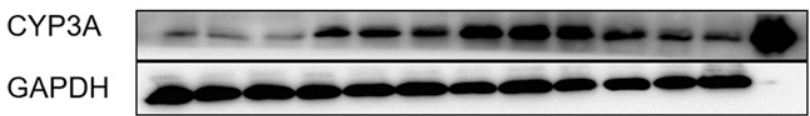

Interaction: $P<0.01$

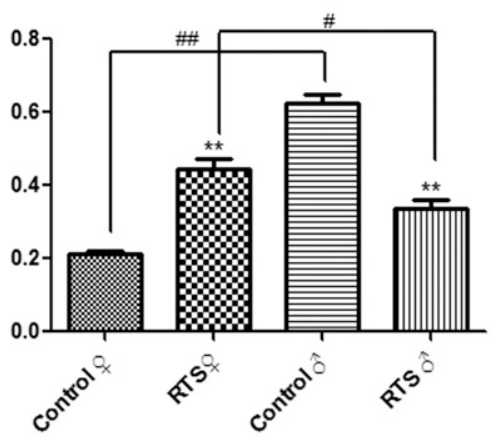

Fig. 7. Effect of RTS on CYP3A expression in rat fetal liver. Pregnant rats were treated with RTS $(20 \mathrm{mg} / \mathrm{kg}$ ) from GD 9-20. Each well was loaded with $50 \mu \mathrm{g}$ of fetal liver protein or $5 \mu \mathrm{g}$ of maternal liver microsomes as positive control. Data are shown as mean \pm S.E.M. for gray density, $n=3$. ${ }^{* *} P<0.01$ vs. control; ${ }^{\#} P<0.05 ;{ }^{\# \#} P<0.01$ vs. female pup. According to two-way analysis of variance (ANOVA) analysis, there was an interaction between fetal gender and RTS treatment on CYP3A protein level. Bonferroni's test was used to identify significant differences among groups.

adduction observed in fetal liver. Some critical detoxifying enzymes, such as glutathione $S$-transferases, are also much less in fetal than that in maternal liver (Wells et al., 2009), and activities of most antioxidative enzymes in embryonic liver are only around $5 \%$ of that in the maternal liver (Wells et al., 2005). In addition, fetuses are more susceptible to genetic damage than adults. After exposure to tobacco smoke for 20 days, fetuses showed greater DNA damage than did the dams (Florek et al., 1998). These factors may contribute to the high susceptibility of a fetus to reactive intermediate-mediated toxicity such as RTS-induced liver injury.

Local metabolic activation seems to be the primary cause of RTSinduced fetal liver toxicity. Juchau and coworkers have mentioned that exported reactive intermediates generated from maternal liver were unlikely to reach embryonic targets and were impossible to pass through chorioallantoic and/or yolk sac membranes from maternal systemic circulation (Juchau, 1989). The present study clearly demonstrated a weak but certain RTS metabolism in normal fetal liver. The weak basal metabolic activation of RTS by fetal liver microsomes in vitro is in agreement with the low level of pyrrole protein adduct generation in pup liver in vivo. Since pyrrole-protein adduct is a definite marker for PA toxicity (Gao et al., 2015; Ruan et al., 2015; Xia et al., 2016), our result suggests that fetal hepatotoxicity may arise from the formation of protein adduction derived from electrophilic intermediates of RTS, formed mainly in situ and mediated by fetal hepatic CYP3A. Moreover, it is well known that placenta is an important pregnancy maintenance tissue and is responsible for nutrients and oxygen exchanges between mother and fetus (Longo and Reynolds, 2010). Fetal growth retardation may happen if the placenta is impaired chronically (Iskender-Mazman et al., 2014). It is likely that PA-induced fetal toxicity in rats partially resulted from placenta injury.

In normal physiologic condition, CYP3A expression in the male liver is higher than in the female liver, both for adult (Lin et al., 2002, 2003, 2007) and fetal rats (Fig. 7). Interestingly, our results showed that RTS caused a sexual dimorphic induction of CYP3A. Apparently, RTS induced hepatic CYP3A expression in the female fetus, whereas the alkaloid attenuated expression of the protein in male fetus, which resulted in the observed gender-related metabolic difference of RTS in fetal rats in vivo (Figs. 6 and 7). Previous study did not show CYP3A induction by a single dose of $30 \mathrm{mg} / \mathrm{kg}$ RTS in male Fischer 344 rats (Gordon et al., 2000), but our results demonstrated an increase of CYP3A protein level in fetal liver after a 12-day exposure to RTS, and we found for the first time that RTS exposure led to a gender-specific induction of CYP3A. Such genderspecific CYP3A induction by other chemical inducers in adult rats was also documented, including methoxychlor, dichlorodiphenyltrichloroethane, pregnenolone $16 \alpha$-carbonitrile, and rifampin (Sierra-Santoyo et al., 2000; Gorski et al., 2003; Oropeza-Hernández et al., 2003; Jan et al., 2006), but the mechanisms of femalespecific induction of CYP3A remain unknown.

In summary, the present study examined the metabolic activity of RTS in maternal-placental-fetal unit in vitro and evaluated embryo toxicity and fetal local metabolic activation of the PA in vivo. The main

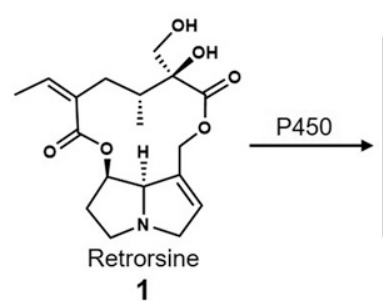

1

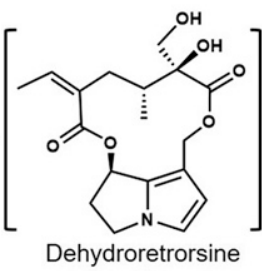

2
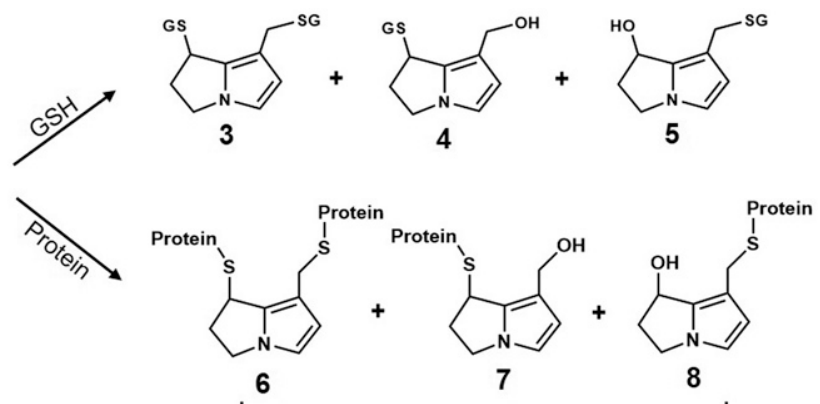
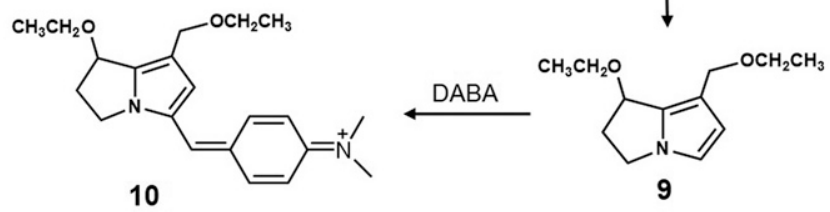

Scheme 1 Proposed formation of pyrrole-GSH conjugates and pyrrole-protein adducts as a result of RTS metabolic activation and synthesis of compound $\mathbf{1 0}$ from pyrroleprotein adducts by chemical derivatization ${ }^{a}$. (a: DABA, 4-dimethylaminobenzaldehyde). 
conclusions included the following points: 1) rat fetal liver and placenta was capable to metabolize RTS; 2 ) prenatal exposure to RTS caused remarkable organ/tissue injury and loss of fetal body weight; 3 ) RTS almost permeated "placenta barrier" completely, but the levels of the resulting pyrrole-protein adduction varied in maternal liver, fetal liver, and placenta; 4) more pyrrole-protein adduction took place in female pups than in male pups, and 5) the observed female-specific metabolic activation of RTS may arise from higher CYP3A expression in female fetal liver, induced by long-term exposure to PAs.

\section{Authorship Contributions}

Participated in research design: Guo, Zheng, Wang.

Conducted experiments: Li, Yang, Xiang, Luo, Qiu, Fang, Zhang.

Performed data analysis: Guo, Yang.

Wrote or contributed to the writing of the manuscript: Li, Yang, Guo, Zheng.

\section{References}

Bhattacharyya K (1965) Foetal and neonatal responses to hepatotoxic agents. J Pathol Bacteriol 90 $151-161$.

Chen M, Li L, Zhong D, Shen S, Zheng J, and Chen X (2016) 9-Glutathionyl-6,7-dihydro-1hydroxymethyl-5H-pyrrolizine is the major pyrrolic glutathione conjugate of retronecine-type pyrrolizidine alkaloids in liver microsomes and in rats. Chem Res Toxicol 29:180-189.

Ejiri N, Katayama K, and Doi K (2003) Induction of CYP3A1 by dexamethasone and pregnenolone-16alpha-carbonitrile in pregnant rat and fetal livers and placenta. Exp Toxicol Pathol 54:273-279.

Fabian E, Wang X, Engel F, Li H, Landsiedel R, and van Ravenzwaay B (2016) Activities of xenobiotic metabolizing enzymes in rat placenta and liver in vitro. Toxicol In Vitro 33:174-179.

Florek E, Tadrowska M, and Szyfter K (1998) Tobacco smoke-induced DNA strand breaks in rat estimated by comet assay. Toxicol Lett $\mathbf{9 5}: 186$.

Gao H, Ruan JQ, Chen J, Li N, Ke CQ, Ye Y, Lin G, and Wang JY (2015) Blood pyrrole-protein adducts as a diagnostic and prognostic index in pyrrolizidine alkaloid-hepatic sinusoidal obstruction syndrome. Drug Des Devel Ther 9:4861-4868.

Gordon GJ, Coleman WB, and Grisham JW (2000) Induction of cytochrome P450 enzymes in the livers of rats treated with the pyrrolizidine alkaloid retrorsine. Exp Mol Pathol 69:17-26.

Gorski JC, Vannaprasaht S, Hamman MA, Ambrosius WT, Bruce MA, Haehner-Daniels B, and Hall SD (2003) The effect of age, sex, and rifampin administration on intestinal and hepatic cytochrome P450 3A activity. Clin Pharmacol Ther 74:275-287.

Guo Y, Ma Z, Kou H, Sun R, Yang H, Smith CV, Zheng J, and Wang H (2013) Synergistic effects of pyrrolizidine alkaloids and lipopolysaccharide on preterm delivery and intrauterine fetal death in mice. Toxicol Lett 221:212-218.

Hakkola J, Pasanen M, Hukkanen J, Pelkonen O, Mäenpää J, Edwards RJ, Boobis AR, and Raunio H (1996a) Expression of xenobiotic-metabolizing cytochrome P450 forms in human full-term placenta. Biochem Pharmacol 51:403-411.

Hakkola J, Raunio H, Purkunen R, Pelkonen O, Saarikoski S, Cresteil T, and Pasanen M (1996b) Detection of cytochrome P450 gene expression in human placenta in first trimester of pregnancy. Biochem Pharmacol 52:379-383.

Hines RN (2008) The ontogeny of drug metabolism enzymes and implications for adverse drug events. Pharmacol Ther 118:250-267.

Huybrechts B and Callebaut A (2015) Pyrrolizidine alkaloids in food and feed on the Belgian market. Food Addit Contam Part A Chem Anal Control Expo Risk Assess 32:1939-1951.

İskender-Mazman D, Akçören Z, Yiğit Ş, Kale G, Korkmaz A, Yurdakök M, and Durukan T (2014) Placental findings of IUGR and non-IUGR. Turk J Pediatr 56:368-373.

Jan YH, Mishin V, Busch CM, and Thomas PE (2006) Generation of specific antibodies and their use to characterize sex differences in four rat P450 3A enzymes following vehicle and pregnenolone 16alpha-carbonitrile treatment. Arch Biochem Biophys 446:101-110.

Juchau MR (1989) Bioactivation in chemical teratogenesis. Annu Rev Pharmacol Toxicol 29: 165-187.

Lankas GR, Wise LD, Cartwright ME, Pippert T, and Umbenhauer DR (1998) Placental P-glycoprotein deficiency enhances susceptibility to chemically induced birth defects in mice. Reprod Toxicol 12:457-463.

Lin G, Cui YY, and Liu XQ (2003) Gender differences in microsomal metabolic activation of hepatotoxic clivorine in rat. Chem Res Toxicol 16:768-774.

Lin G, Cui YY, Liu XQ, and Wang ZT (2002) Species differences in the in vitro metabolic activation of the hepatotoxic pyrrolizidine alkaloid clivorine. Chem Res Toxicol 15:1421-1428.

Lin G, Tang J, Liu XQ, Jiang Y, and Zheng J (2007) Deacetylclivorine: a gender-selective metabolite of clivorine formed in female Sprague-Dawley rat liver microsomes. Drug Metab Dispos 35:607-613.
Lin G, Wang JY, Li N, Li M, Gao H, Ji Y, Zhang F, Wang H, Zhou Y, Ye Y, et al. (2011) Hepatic sinusoidal obstruction syndrome associated with consumption of Gynura segetum. J Hepatol 54: $666-673$.

Longo LD and Reynolds LP (2010) Some historical aspects of understanding placental development, structure and function. Int J Dev Biol 54:237-255.

Maezawa K, Matsunaga T, Takezawa T, Kanai M, Ohira S, and Ohmori S (2010) Cytochrome P450 3As gene expression and testosterone 6 beta-hydroxylase activity in human fetal membranes and placenta at full term. Biol Pharm Bull 33:249-254.

Medeiros RM, Górniak SL, and Guerra JL (2000) Fetotoxicity and reproductive effects of monocrotaline in pregnant rats. J Ethnopharmacol 69:181-188.

Oropeza-Hernández LF, López-Romero R, and Albores A (2003) Hepatic CYP1A, 2B, 2C, 2E and 3 A regulation by methoxychlor in male and female rats. Toxicol Lett 144:93-103.

O'Shaughnessy PJ, Monteiro A, Bhattacharya S, and Fowler PA (2011) Maternal smoking and fetal sex significantly affect metabolic enzyme expression in the human fetal liver. J Clin Endocrinol Metab 96:2851-2860.

Panter KE, Gardner DR, and Molyneux RJ (1998) Teratogenic and fetotoxic effects of two piperidine alkaloid-containing lupines (L. formosus and L. arbustus) in cows. J Nat Toxins 7: 131-140.

Peterson JE and Jago MV (1980) Comparison of the toxic effects of dehydroheliotridine and heliotrine in pregnant rats and their embryos. $J$ Pathol 131:339-355.

Radominska-Pandya A (2010) Preface. Drug Metab Rev 42:1-2.

Roulet M, Laurini R, Rivier L, and Calame A (1988) Hepatic veno-occlusive disease in newborn infant of a woman drinking herbal tea. J Pediatr 112:433-436.

Ruan J, Gao H, Li N, Xue J, Chen J, Ke C, Ye Y, Fu PP, Zheng J, Wang J, et al. (2015) Blood pyrrole-protein adducts-a biomarker of pyrrolizidine alkaloid-induced liver injury in humans. $J$ Environ Sci Health C Environ Carcinog Ecotoxicol Rev 33:404-421.

Sandini TM, Udo MS, Reis-Silva TM, Bernardi MM, and Spinosa HdeS (2014) Prenatal exposure to integerrimine $\mathrm{N}$-oxide impaired the maternal care and the physical and behavioral development of offspring rats. Int J Dev Neurosci 36:53-63.

Sierra-Santoyo A, Hernández M, Albores A, and Cebrián ME (2000) Sex-dependent regulation of hepatic cytochrome P-450 by DDT. Toxicol Sci 54:81-87.

Smith LW and Culvenor CC (1981) Plant sources of hepatotoxic pyrrolizidine alkaloids. J Nat Prod 44:129-152.

Sundareson AE (1942) An experimental study on placental permeability to cirrhogenic poisons. $J$ Pathol Bacteriol 54:289-298.

Tu M, Li L, Lei H, Ma Z, Chen Z, Sun S, Xu S, Zhou H, Zeng S, and Jiang H (2014) Involvement of organic cation transporter 1 and CYP3A4 in retrorsine-induced toxicity. Toxicology 322: $34-42$.

Wang YP, Fu PP, and Chou MW (2005) Metabolic activation of the tumorigenic pyrrolizidine alkaloid, retrorsine, leading to DNA adduct formation in vivo. Int J Environ Res Public Health 2 : 74-79.

Wells PG, Bhuller Y, Chen CS, Jeng W, Kasapinovic S, Kennedy JC, Kim PM, Laposa RR, McCallum GP, Nicol CJ, et al. (2005) Molecular and biochemical mechanisms in teratogenesis involving reactive oxygen species. Toxicol Appl Pharmacol 207 (2, Suppl):354-366.

Wells PG, McCallum GP, Chen CS, Henderson JT, Lee CJ, Perstin J, Preston TJ, Wiley MJ, and Wong AW (2009) Oxidative stress in developmental origins of disease: teratogenesis, neurodevelopmental deficits, and cancer. Toxicol Sci 108:4-18.

Wyde ME, Kirwan SE, Zhang F, Laughter A, Hoffman HB, Bartolucci-Page E, Gaido KW, Yan B, and You L (2005) Di-n-butyl phthalate activates constitutive androstane receptor and pregnane $X$ receptor and enhances the expression of steroid-metabolizing enzymes in the liver of rat fetuses. Toxicol Sci 86:281-290.

Xia Q, Zhao Y, Lin G, Beland FA, Cai L, and Fu PP (2016) Pyrrolizidine alkaloid-protein adducts: potential non-invasive biomarkers of pyrrolizidine alkaloid-induced liver toxicity and exposure. Chem Res Toxicol 29:1282-1292.

Yang X, Li W, Sun Y, Guo X, Huang W, Peng Y, and Zheng J (2017) Comparative study of hepatotoxicity of pyrrolizidine alkaloids retrorsine and monocrotaline. Chem Res Toxicol 30: $532-539$.

Yang YC, Yan J, Doerge DR, Chan PC, Fu PP, and Chou MW (2001) Metabolic activation of the tumorigenic pyrrolizidine alkaloid, riddelliine, leading to DNA adduct formation in vivo. Chem Res Toxicol 14:101-109.

Zhou SF, Xue CC, Yu XQ, and Wang G (2007) Metabolic activation of herbal and dietary constituents and its clinical and toxicological implications: an update. Curr Drug Metab 8: 526-553.

Address correspondence to: Yu Guo, Department of Pharmacology, School of Basic Medical Science, Wuhan University, Wuhan 430071, P. R. China; Hubei Provincial Key Laboratory of Developmentally Originated Disease, Wuhan 430071, P. R. China. E-mail: guoy@whu.edu.cn; or Jiang Zheng, Wuya College of Innovation, Shenyang Pharmaceutical University, Shenyang, Liaoning 110016, P. R. China; State Key Laboratory of Functions and Applications of Medicinal Plants, Key Laboratory of Pharmaceutics of Guizhou Province, Guizhou Medical University, Guiyang, Guizhou 550004, P. R. China. E-mail: zhengneu@yahoo.com 